\title{
PHOTO-ESR STUDY OF THE DX TO SHALLOW DONOR CONVERSION IN Te DOPED $\mathrm{Al}_{x} \mathrm{Ga}_{1-x} \mathrm{As}$
}

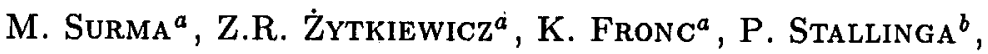 \\ AND M. GODLEWSKI ${ }^{a}$ \\ ${ }^{a}$ Institute of Physics, Polish Academy of Sciences \\ Al. Lotników 32/46, 02-668 Warszawa, Poland \\ ${ }^{b}$ Van der Waals-Zeeman Lab., Amsterdam University \\ Valckenierstraat 65, 1018 XE Amsterdam, The Netherlands
}

\begin{abstract}
Results of detailed electron spin resonance (ESR) study of Te doped $\mathrm{Al}_{x} \mathrm{Ga}_{1-x}$ As epilayers with $x=0.41,0.42$, and $0.5 \mathrm{Al}$ fractions are presented. It is shown that the ESR signal observed critically depends on cooling steps and that the shallow donor ESR signal can be observed prior to illumination. The first ESR study of AlGaAs layers with removed GaAs substrate are presented. The mechanism of the enhanced photosensitivity of the ESR signal is explained. It is found very paradoxical that the ESR signals decreases upon the illumination even though shallow donor concentration is increased.
\end{abstract}

PACS numbers: 71.55.Eq, 76.30.Lh, 78.66.Fd

\section{Introduction}

The so-called DX state of group IV and group VI donors in $\mathrm{Al}_{x} \mathrm{Ga}_{1-x} \mathrm{As}$ is the most studied deep impurity state in common semiconductors [1]. Application aspects caused that most of the studies were performed for Si doped layers. Here we present electron spin resonance (ESR) of liquid phase epitaxy (LPE) grown samples and the first study performed on thick liquid phase electroepitaxy (LPEE) grown AlGaAs epilayer with removed GaAs substrate. These measurements were performed on heavily Te doped $\mathrm{Al}_{x} \mathrm{Ga}_{1-x} \mathrm{As}(x=0.41)$ epilayers. The details on growth procedure and experimental set up can be found elsewhere $[2,3]$. An annoying situation is reported that the ESR, optically detected magnetic resonance (ODMR) and photo-ESR investigations can misleadingly "verify" often conflicting models of the DX centers. 


\section{ESR study}

Lack of the ESR signal of the DX state was a major argument for the negative- $U$ model of DX state [4]. Our present results show that one should be very careful in accepting this argument. In Fig. 1 we show ESR spectra observed for

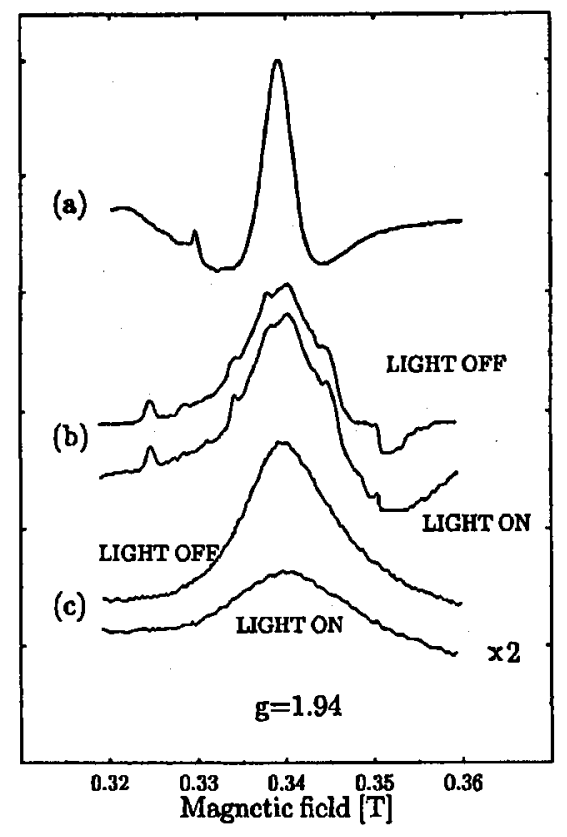

Fig. 1. Electron spin resonance spectra of LPE grown $x=0.42$ (a) and $x=0.5(\mathrm{~b})$ $\mathrm{Al}_{x} \mathrm{Ga}_{1-x}$ As layers. The spectra shown in (b) were measured before and after the illumination. The ESR spectrum shown in (c) was measured for $x=0.41$ LPEE layer after removing the GaAs substrate. The ESR signal shown was observed for a sample cooled down to $4 \mathrm{~K}$ temperature in total darkness before and after $120 \mathrm{~s}$ of white light illumination.

a range of different $\mathrm{Al}_{x} \mathrm{Ga}_{1-x}$ As layers: lightly Te doped $\left(10^{16} \mathrm{~cm}^{-3}\right)$ LPE grown sample with $x=0.42$ (a), and $x=0.5$ (b), and heavily doped LPEE sample with $x=0.41$ (c). The two latter spectra were recorded for a sample cooled down in total darkness. The LPE samples were too thin to remove the GaAs substrate. The ESR of $x=0.42 \mathrm{LPE}$ sample shows a broad resonance signal at $g=2$ in dark. A new signal appears under intensive white light illumination (Fig. 1). This photosensitive signal could be observed for samples (b) and (c) also without any illumination. Moreover, its magnitude was decreased by the illumination in the sample (c), in contrary to the results found for the sample (a).

Similar ESR signal was observed previously in low sensitivity (thin MOVPE sample was studied) ESR investigations of Bardeleben and coworkers $[4,5]$ and was attributed to the ESR signal of shallow Te donor state. The identification was 
based on photosensitivity of the signal and on its metastable behavior after turning off the illumination at low temperature. We report here that the above arguments are not sufficient. Photoexcitation of high mobility $\mathrm{Al}_{x} \mathrm{Ga}_{1-x} \mathrm{As}$ resulted for all samples studied in large decrease in the $Q$-factor of microwave cavity. This affects magnitude of all ESR signals observed and accounts for nearly $50 \%$ decrease in ESR signal of carbon probe sample mounted with thick LPEE layer. The decrease of the ESR signals was metastable at low temperature. Thus, $g=2$ and $g=1.94$ signals, but carbon probe sample as well, had properties expected for ESR spectra of shallow Te donor in $\mathrm{Al}_{x} \mathrm{Ga}_{1-x}$ As. Photosensitivity and metastable character of light induced changes are, thus, not sufficient argument to determine which of the ESR signals, if any, is Te-related. The situation was still less clear for the results obtained for the $x=0.5$ sample. Here $g=1.94$ signal could consistently be observed for the sample cooled down in dark and only small effect of white light excitation was observed (Fig. 1).

In this work. we base the identification on two separate studies. First, on optically detected magnetic resonance experiments [6] and on ESR investigations of thick LPEE layers with removed GaAs substrate. The ODMR investigations prove that $g=2$ signal is related to GaAs substrate. The identity of $g=1.94$ signal is less clear. This signal was observed via the donor-acceptor pair (DAP) emission of AlGaAs, but the best condition for its detection was via DAP processes of GaAs [6]. The latter is due to very efficient energy transfer link between epilayer and substrate. The efficient energy transfer link causes that the identity of $g=1.94$ spectrum could not be unambiguously explained by the ODMR experiment. In this sense the ESR study of thick $\mathrm{Al}_{x} \mathrm{Ga}_{1-x}$ As layers before and after removing the GaAs substrate was expected to be concluding. However, the first results of the ESR experiments were very unexpected [7]. The $g=1.94$ signal was observed in dark and its magnitude was decreased by above $0.65 \mathrm{eV}$ illumination (Fig. 1). This is why the signal was first attributed to some unidentified deep defect of AlGaAs and not to Te-related shallow donor state [7].

The detailed studies of signal properties show, however, that all its properties are consistent with ESR spectrum of Te shallow donor state. Donor conversion to the deep DX state is always partial even for the sample cooled down in total darkness [8]. For heavily Te doped sample, concentration of inequilibrium shallow donor state can be above ESR detection limit. This is why we could observe the relevant ESR signal before any illumination. However, due to the same reason the sample can remain conducting, since shallow donor concentration may remain above the Mott limit, i.e., it is above about $10^{17} \mathrm{~cm}^{-3}[5,9]$. The latter is confirmed by the present experimental results. The ESR signal of LPEE sample was asymmetric in shape of the Dyson type. Using a carbon probe sample, we found that sample illumination increases conductivity and the observed decrease in the ESR signals follows the decrease in the cavity $Q$-factor. The effect is proportional to sample conductivity and leads to paradoxical situation of decreasing ESR signal of shallow Te donor even though the concentration of the donors increases upon illumination. We utilized the effect to perform detailed photo-ESR investigations of DX-shallow donor conversion processes induced either by illumination or by sample heating. These photo-ESR experiments reproduced well-known spectra 
measured previously with other methods and it will not be discussed in detail here.

The controversial aspect of recent DX investigations was the sequence of DX ionization. It was proposed that the DX ionization is a two-step process proceeding via localized state of Te neutral donor [10]. Two-exponential kinetics was expected in this case. This was verified in our ESR study. Changing initial concentration of shallow Te donor (by changing the cooling speed of the sample), we could observe either two-exponential or purely one-exponential kinetics of the photosensitivity of ESR signal. The observation is consistent with rate equations describing two step DX ionization. We indicate, however, that for heavily doped sample the one-exponential kinetics is observed in most of the cases, which could be used to reject a two-step ionization process of DX-conduction band transition.

Finally, we report that DX ionization is most efficient for illumination creating free holes in the sample.

\section{References}

[1] P.M. Mooney, J. Appl. Phys, 67, R1 (1990).

[2] Z.R. Żytkiewicz, S. Miotkowska, J. Cryst. Growth 121, 457 (1992).

[3] M. Surma, Z.R. Żytkiewicz, M. Godlewski, to be published.

[4] H.J. von Bardeleben, Solid State Phenom. 10, 181 (1989).

[5] H.J. von Bardeleben; M. Zazoui, S. Alaya, P. Gibart, Phys. Rev. B 42, 1500 (1990).

[6] M. Godlewski, K. Fronc, W.M. Chen, B. Monemar, Acta Phys. Pol. A 80, 341 (1991).

[7] M. Surma, M. Godlewski, Z.R. Żytkiewicz, Acla Phys. Pol. A 82, 817 (1992).

[8] P.W.M. Blom, P.M. Koenraad, F.A.P. Blom, J.H. Wolter, J. Appl. Phys. 66, 4269 (1989).

[9] P. Galtier, G. Martinez, B. Lambert, M. Gauneau, Phys. Rev. B 33, 6909 (1986).

[10] L. Dobaczewski, P. Kaczor, Phys. Rev. B 44, 8621 (1991). 